\title{
TO RETURN OR TO DISCARD?. RANDOMISED TRIAL ON GASTRIC RESIDUAL VOLUME MANAGEMENT
}

Juvé-Udina, Maria-Eulàlia ${ }^{a^{*}}, \mathrm{RN}, \mathrm{MSN}$

Valls-Miró, Consol ${ }^{\mathrm{b}}$, RN, BS

Carreño-Granero, Avelina ${ }^{\mathrm{b}}$, RN

Martinez-Estalella, Gemma ${ }^{\text {b }}$, RN

Monterde-Prat, David ${ }^{\mathrm{a}}$, BSS

Domingo-Felici, Carmen-Maria ${ }^{\mathrm{b}}$, RN

Llusa-Finestres, Joan ${ }^{\mathrm{b}}$, RN

Asensio-Malo, Gemma ${ }^{\mathrm{b}}$, RN

a IDIBELL, Catalan Institute of Health. Gran Via de les Corts Catalanes, 587, Barcelona 08007, Spain

${ }^{b}$ IDIBELL, Bellvitge University Hospital, ICU 2-1, Feixa Llarga s/n. 08907 Hospitalet de Llobregat, Spain

email addresses: gemma@bellvitgehospital.cat ; ejuve@gencat.cat

* Corresponding author. Tel. +34 675786360 Fax +34 934824205

KEYWORDS

Gastric residual volume; nasogastric tube; critical care; clinical trial; nursing 


\section{SUMMARY}

Background: The control of gastric residual volume (GRV) is a common nursing intervention in intensive care; however the literature shows a wide variation in clinical practice regarding the management of GRV, potentially affecting patients' clinical outcomes.

The aim of this study is to determine the effect of returning or discarding GRV, on gastric emptying delays and feeding, electrolyte, and comfort outcomes in critically ill patients.

Method: A randomised, prospective, clinical trial design was used to study 125 critically ill patients, assigned to the return or the discard group. Main outcome measure was delayed gastric emptying. Feeding outcomes were determined measuring intolerance indicators, feeding delays and feeding potential complications. Fluid and electrolyte measures included serum potassium, glycaemia control and fluid balance. Discomfort was identified by significant changes in vital signs.

Results: Patients in both groups presented similar mean GRV with no significant differences found $(p=0,111)$, but participants in the intervention arm showed a lower incidence and severity of delayed gastric emptying episodes $(p=0,001)$. No significant differences were found for the rest of outcome measurements, except for hyperglycaemia.

Conclusions: The results of this study support the recommendation to reintroduce gastric content aspirated to improve GRV management without increasing the risk for potential complications. 


\section{INTRODUCTION}

In healthy adults, the gastrointestinal (GI) tract produces seven to nine litres of secretions daily. Of these secretions, "most are absorbed in the small bowel and about 500 to $600 \mathrm{ml}$ reaches the colon, where another $350 \mathrm{ml}$ is absorbed; $150 \mathrm{~g}$ of stool remains" (Jeejeebhoy, 2002; Jeejeebhoy, 1977).

The rate of gastric emptying is regulated by neural and humoral mechanisms and in fasting conditions, retention of 10 to $100 \mathrm{ml}$ of fluid gastric content can be considered physiological (Edwards, 2000; McClave, 1992).

Delayed gastric emptying is common in critically ill patients, occurring in approximately $50 \%$ of mechanically ventilated individuals. This may be due to impaired gastroduodenal motility related to patients' clinical severity, premorbid conditions, and pharmacological and surgical treatments. Prevention and management of delayed gastric emptying include the insertion of a nasogastric tube (NGT) to aspirate gastric contents. (Deane et al., 2007; Dive et al., 1994;

\section{Ritz et al., 2001, Stechmiller et al., 1997).}

Enteral feeding (EF) is considered the preferred method of nutritional support for the critically ill. EF offers nutritional advantages, contributes to bowel flora maintenance, reduces infection risks, and avoids the potential adverse outcomes of parenteral nutrition. However, it is not exempt from complications (pulmonary aspiration, tube occlusions or intolerance). In this population, GI dysmotility implies feeding via a NGT is often associated with large gastric residual volumes, which may lead to increase the potential for regurgitation and vomiting (McClave et al., 1992; Edwards \& Metheny, 2000; Williams and Leslie,

2004) and a delay in the achievement of nutritional goals, because of under- 
delivery of feeds (Engel, 2003; Marshall and West, 2006; McClave, 1999; Pingleton, 2001).

A common nursing intervention to assess gastrointestinal function and to minimise potential complications from enteral nutritional therapy (ENT) in critically ill patients, is the regular checking of gastric residual volume (GRV) by aspiration with a syringe. But like in many other clinical procedures, standards for the management of GRV are not evidence-based. Reports in the literature show a wide variation in nursing practices, regarding the frequency of GRV checks, whether to instil gastric contents obtained to the patient or to discard volume aspirated, and what should be considered maximum GRV (Goodwin, 1996; Metheny, 1993; Mori et al., 2003; Pullen 2004; Valls et al., 2006; Williams and Leslie, 2004). Thus, based on empirical observations and clinical experience, authors have proposed maximum GRV for critically ill patients between 50 and $500 \mathrm{ml}$ (Marshall and West, 2006; McClave, 2002; Metheny, 2004; Murphy 1999).

A different approach to GRV management is emerging. High gastric residual volumes is not always indicative of gastric stasis (Burt and Lentz, 2001; Jostee et al., 1999; McClave and Snider, 2002, Williams and Leslie, 2004), a low GRV does not protect against aspiration pneumonia (Lukan et al., 2002; McClave and Snider, 2002; Pinilla et al., 2001; Powell et al., 1993, Williams and Leslie,

2004) and as stated by Zaloga (2005), "the level at which GRV predicts aspiration risk still remains unknown". While the scientific community is trying to respond the utility of the bedside measurement of GRV (Chang et al., 2007; Zaloga, 2005), some nurses discard gastric contents while others reintroduce it 
to the patient, partially or completely, depending on their assessment al., 2000; Marshall and West, 2006; Mateo, 1996). Individual beliefs, unit tradition, expert opinion or nurse's experience guide the decision. Some authors support instilling gastric content aspirated in order to contribute to the maintenance of gastric juices and the electrolyte balance. Others hypothesise discarding as the best option in order to avoid tube contamination, infection risks, tube complications like occlusion, as well as to prevent volume retention secondary to delayed gastric emptying (Booker et al., 2000).

\section{Objectives}

Authors in this inquiry looked for responding the clinical question: what is better for the patient, to return or to discard the GRV aspirated?.

The main purpose of this study was to investigate, in adult critically ill patients, the effects of returning versus discarding gastric residual volume on gastric volume and gastric emptying delays.

Specific goals included to evaluate whether the gastric residual volume determination with the GRV reintroduction technique augments the incidence of:

(1) NGT obstructive complication episodes; (2) pulmonary aspiration episodes;

(3) intolerance episodes (nausea, vomiting, diarrhoea and abdominal distension); (4) enteral feeding delays; (5) hyperkalaemia episodes; (6) hyperglycaemia episodes and (7) discomfort episodes, when compared to the discarding group. 


\section{METHOD}

\section{Study design}

This prospective, randomised, non-blinded, clinical trial was conducted over one year period, in a single medical-surgical intensive care unit (ICU) of a public university hospital. Institutional ethics committee approval was granted and informed consent was obtained from the patient or a relative legally authorised.

\section{Participants}

Any patient admitted to the ICU, aged 18 or more, haemodynamically monitored, enterally or parenterally fed, all of them needing GRV controls due to their condition and treatment, with a length of stay estimated at greater than 48 hours, was considered eligible to enter the study.

Exclusion criteria included patients connected to an intermittent gastric aspiration system because of paralytic ileum, bowel obstruction, gastric fistula or gastric surgery.

A computer-generated random list and sealed envelope were used to randomise patients to the GRV return group or the GRV discard arm. It was calculated that using an alpha of $0.05,59$ patients would need to be entered in each group to have an $80 \%$ power of detecting a difference greater than of $10 \%$ in the rate of delayed gastric emptying episodes.

\section{Data Collection}

Each subject remained in the study until any of the following end points was met: no need for further GRV controls (medical indication of NGT withdrawal), critical adverse event associated to the procedure (pulmonary aspiration or 
cardio respiratory arrest during or immediately after the procedure), transferring out of ICU, faecal aspirates, major protocol error, or death.

All data were obtained by the primary investigators or by the trained registered nurses from the ICU.

Gastric residual volumes were checked, as usual in the unit, every six hours, aspirating the content through a $60 \mathrm{ml}$ syringe. Stomach was considered aspirated to dryness when no more gastric content could be obtained with the aspiration syringe. No position changes were performed during the GRV controls. Research team and the medical staff determined that GRV would be instilled to patients in the return group, up to a maximum of $250 \mathrm{ml}$ per check. If volume obtained from aspiration was greater than $250 \mathrm{ml}$, surplus from that cutting point was discarded. Exact measurement of the amount aspirated and returned was recorded in the patients' data collection forms.

Gastric emptying delay (GED) was defined as the difficulty in maintaining gastric residual volume within safe limits (GRV below $5 \mathrm{ml} / \mathrm{kg}$ ) (Horn et al. 2004). Based on the available evidence, it was categorised as light GED (151 $250 \mathrm{ml} / 6 \mathrm{~h})$, moderate GED (251 - $350 \mathrm{ml} / 6 \mathrm{~h})$ or severe GED (> $350 \mathrm{ml} / 6 \mathrm{~h})$. All patients in the study had head-of-bed kept at $30^{\circ}$ or greater. Feeding rhythm (delays) in patients with ENT was controlled through the determination of the difference $(>20 \%)$ between the amount prescribed and the amount administered every $24 \mathrm{~h}$. Administration of the formula was continuous and controlled by a pump delivery system. ENT was temporally withheld if any of the following conditions were present: GRV greater than $500 \mathrm{ml}$ (Figure 1); 
vomiting; need of radiological or surgical procedures and diarrhoea for more than 48 hours.

Nasogastric tube was checked for occlusion every shift. Prescribed formulas administered and standards for NGT care and maintenance, followed by all nursing staff, are detailed in Figure 2.

Daily scheduled lab test (7 a.m.) was obtained for serum potassium, sodium, glycaemia and proteins. Hypokalaemia was defined as a blood potassium value $<3.5 \mathrm{mmol} / \mathrm{L}$ in the 7 a.m. lab test. Hyperglycaemia as blood glucose value $>8$ $\mathrm{mmol} / \mathrm{L}$ and hypoalbuminemia considered when albumin was $<0.54 \mathrm{mmol} / \mathrm{L}$. Fluid balance was calculated partially, every shift and at 8 a.m. on a 24-hour basis.

Before and after each GRV check, vital signs and Ramsey Sedation Scale (Ramsay MA et al., 1974) were assessed to identify any signs of discomfort in the patient due to the procedure.

Patients were prospectively monitored for nausea, vomiting, abdominal distension (checking abdominal circumference following standardised procedure every 24 hours), diarrhoea (three or more soft blob-like, mushy or liquid stools / 24 hours) (Booker et al., 2000; Lewis and Heaton KW, 1997) and pulmonary aspiration. For patients with artificial airway, the standard blood glucose monitors to check pulmonary aspirates were prepared to use. Investigators also gather data on patients' medication, especially prokinetics and drugs affecting gastric emptying along with other general data of interest. 


\section{Data analysis}

Sample characteristics and main outcomes were analysed by using frequencies, measures of central tendency, Wilcoxon test, chi square or pair sample $t$ test as appropriate. Univariate analysis of variance (ANOVA) was use to analyse the factors contributing to residual volume.

To assess the adequacy of randomisation, groups were compared by the chisquare test when appropriate, Fisher's exact test for categorical variables, and Wilcoxon or Kruskal-Wallis tests for continuous variables.

Significance was established at an alpha value of 0.05 . Descriptive statistics were used to report complications.

All analysis was made using $R$ release 2.3.1 (R Development Core team, 2006).

\section{RESULTS}

We finally recruited 125 consecutive patients; 63 were randomly assigned to the intervention (return) group and 62 to the control (discard) group. Two patients in the intervention arm and one assigned to the control group could not be included in the final analysis because of a shorter than expected length of stay and a major protocol error (has GRV returned) (Figure 3). Overall, 61 patients had their GRV discarded and 61 returned.

The baseline features of the patients in each group were similar and no significant differences were found with regard to age, sex, primary and secondary diagnosis, APACHE II and NEMS scores, mechanical ventilation and enteral feeding (Table 1). 
Patients in the intervention group showed a slightly lower total mean GRV although this difference has no statistical significance $(p<0.111)$ (Table 2$)$. This result was consistent through time comparisons and when adjusting through ANOVA model to confusion variables: hyperglycaemia, prokinetics and medication delaying gastric emptying, including opiates, atropine, barbiturates and neuromuscular blocking agents $(F=0.847)(\operatorname{Pr}(>F) 0.359)$. Mean ratio of gastric content reintroduction in the intervention group was 0.93 (SD 0.25). GRV was not related to diagnosis, demographic variables or to APACHE II score.

Incidence and severity of delayed gastric emptying (GED) episodes were lower in the intervention group $(p=0.001)$. The number of light and moderate GED episodes was double in the discard group (Table 2). Severe GED episodes were observed both in patients feed enterally and those with parenteral nutrition and were also more frequent in the control group.

No differences were found between groups in the number of patients with ENT and the mean prescribed volume or the administered EF volume. The type of feeding formula and NGT (Salem tube_16 G) were comparable for the two study groups. NGT remained in place while the patients were in the study, for similar mean duration. Episodes of tube blockage and accidental extubation did not occur. Neither statistically significant difference in the number of ENT days was detected, although the mean was slightly greater in the control group. Incidence of a difference greater than $20 \%$ between the amount of ENT prescribed and the volume administered was similar in both groups ( $p=0.91)$ (Table 3). 
Fluid balance and serum electrolyte outcomes were comparable in both groups with no statistically significant differences, except for hyperglycaemic episodes, which were more frequent in the intervention group $(p=0.001)$. Episodes of hyperglycaemia correlate with a higher frequency of moderate and severe GED despite of the group $(p=0.001)$. More patients presented hypokalaemia at any moment of the study in the control group, although this difference was not statistically significant $(p=0.611)$. The number of hypokalaemic episodes distributed similarly in the two groups (Table 4).

For any of the statistical tests applied, no differences were identified between the study groups in relation to the discomfort outcomes. Mean pulse, blood pressure and Ramsey measurements resulted homogeneous with a slightly increase of BP in the intervention group. Neither difference was observed in the comparison of the vital signs measurements before and after the intervention. No significant differences were found for the other outcome measures and the overall rate of complications was similar in both groups (Table 5). Complication rates were null for nausea and pulmonary aspiration and insignificant for vomiting. Four percent of patients more in the intervention group presented diarrhoea at any time of the study, although this value has no statistical significance $(p=0.709)$. Mean days with diarrhoea was similar in both groups. Mean GRV was lower in patients with diarrhoea despite the group, when compared to patients who did not present diarrhoea $(p=0.003)$. All patients presenting diarrhoea were fed with enteral nutrition.

Abdominal distension was documented in more patients from the control group, with no statistical difference observed $(p=0.071)$. 


\section{DISCUSSION}

In the light of these results, reintroducing the gastric content aspirated, up to $250 \mathrm{ml}$ per check, does not increase the number or the severity of complications. Half the patients in the intervention group presented light or moderate GED and less participants suffered from severe GED when compared to the control. This could suggest that reintroducing the GRV aspirated does not increase the total GRV and could have an effect in maintaining GRV at closer physiological levels. This result correlates with the recommendation of returning gastric aspirate to patients (Lin and Van Citters, 1997; McClave et al., 2002; Williams and Leslie, 2005). As stated by Jooste et al. (1999), "having high residual gastric volumes does not always imply gastric stasis". When GRV is higher than $250 \mathrm{ml}$ clinicians should be alerted to potential complications and should activate careful bedside monitoring to manage fluid, nutritional and electrolyte outcomes. ENT delivered via nasojejunal tube has also shown promising results and may be other forms to improve GRV management

\section{(Davies et al., 2002).}

Our results coincide with Van Der Voort's (2001) in detecting no relationship between GRV and APACHE II score.

The absence of tube blockages in any group does not correlate with the results of Booker et al. (2000). The recommendation of regularly flushing the NGT with water (Williams and Leslie, 2005) was followed by nurses in the unit. This fact may have contributed to prevent this complication. In contrast, our results do 
correlate with Booker's (2000) in relation to an equal distribution of feeding delays between both groups.

Patients were kept at safety position (head-of-bed at $30^{\circ}$ elevation) and continuous ENT delivery via peristaltic pump assured constant delivery of small volumes in the stomach (Stevens et al., 2002). These factors have probably contributed to reduce aspiration risk.

Some authors (De Boer et al., 1992; Mallampalli et al., 2000; Zhao et al., 2006) described the important role of hyperglycaemia in oesophageal motility, decreasing inferior oesophageal sphincter pressure, the speed of the oesophageal peristalsis, and in the delay of gastric emptying. In this sense, a trend has been found on detecting a higher number of hyperglycaemic episodes in patients in the intervention group and a relation between moderate and severe GED and hyperglycaemia despite the group.

Discarding gastric aspirate may result in loss of gastric fluids and electrolytes (Cataldi-Betcher et al., 1983), but this hypothesis cannot be verified with our work as we could not identify any differences.

It has been stated that "the uncertainty existing with regard to the measurement, interpretation and management of GRV influences nursing practice" (Williams and Leslie, 2005). This randomised control trial could contribute to diminish this uncertainty but it is not exempt from some significant limitations. Due to the features of the intervention, blinding was not feasible. Although the sample size was appropriate for the research question, larger sample sizes would procure a higher level of evidence and a multi-centre approach could be more appropriate. 
The authors have not investigated the role of ICU nurses' expertise on the prevention and management of potential complications. Care standards were properly applied in the unit; however, the data collection period excluded 2 months in summer, when nursing turnover is higher and probably, less skilled registered nurses are working. This could introduce potential bias in the results. The authors agree with Booker et al. (2000) in that the low incidence of serious complications could be related to proficient nursing care provided, but it should be empirically proved.

Limiting the reintroduction of GRV up to a maximum of $250 \mathrm{ml}$ per check can be considered another important limitation. Results are based on instilling this amount to patients; and the probable outcomes if the total GRV would be returned, can only be inferred, although the mean percentage of volume reintroduction was greater than $90 \%$.

In this study, all patients regardless the group, received 40mg of Omeprazole per $24 \mathrm{~h}$, but this was a standard for any patient admitted in the ICU. Additional administrations or other acid inhibiting agents administration were not controlled. These drugs are described to inhibit the gastric secretion with the corresponding decrease of the GRV (Chang et al., 2007).

Difficulties have also been found in identifying an instrument to measure discomfort in critically ill patients. In the absence of a validated tool, the literature suggested authors to use Ramsey sedation scale and blood pressure as these parameters are usually controlled in this population as indicators of the need for analgesia, sedation and the severity of pain and discomfort. The 
necessary continuous haemodynamical management of critical patients could have masked the results.

Finally, some authors have stated that reintroducing GRV aspirated could precipitate infections due to manipulation (Booker et al., 2000; Pingleton et al., 1986; Williams and Leslie, 2005). In this study we have not been able to gather data on microbiological results; but ICU physicians considered that diarrhoea was not attributable in any of the patients to infection or contamination of the gastric tube. Diarrhoea was not controlled for antibiotic intolerance and this could also be considered a significant limitation of the study.

\section{CONCLUSIONS}

In conclusion, while better evidence is going to be produced by the scientific community, the results of this study support the recommendation to reintroduce gastric aspirate (up to $250 \mathrm{ml}$ per check) in critically ill patients to procure a more physiological gastric content management approach without increasing the risk of severe potential complications, while controlling for glycaemia. 


\section{REFERENCES}

Booker KJ, Niedringhaus L, Eden B, Arnold JS. Comparison of 2 methods of managing gastric residual volumes from feeding tubes. Am J Crit Care 20009 (5): $318-24$

Burd R, Lentz C. The limitations of using gastric residual volumes to monitor enteral feedings: a mathematical model. Nutr Clin Pract 2001; 16 (6): 349-54

Cataldi-Betcher EL, Seltzer MH, Slocum BA, Jones KW. Complications occurring during enteral nutrition support: A prospective study. J Parent Enteral Nutr 19837 (6): 546-52

Chang WK, McClave SA, Hsieh CB, Chao YC. Gastric residual volume and gastric contents measurement by refractometry. J Parent Enteral Nutr 200731 (1): $63-8$

Davies AR, Froomes P, French CJ, Bellomo R, Gutteridge GA, Nyulasi I et al. Randomised comparison of nasojejunal and nasogastric feeding in critically ill patients. Crit Care Med 200230 (3): 586-90 
De Boer SY, Masclee AA, Lam WF, Lamers CB. Effect of acute hyperglycemia on esophageal motility and lower esophageal sphincter pressure in humans. Gatroenterology 1992103 (3): 775-80

Deane A, Chapman MJ, Fraser RJ, Bryant LK, Burgstad C, Nguyen NQ. Mechanisms underlying feed intolerance in the critically ill: Implications for treatment. World J Gastroenterol 2007; 13 (29): 3909-17

Dive A, Moulart M, Jonard P, Jamart J, Mahieu P. Gastroduodenal motility in mechanically ventilated critically ill patients. Crit Care Med 1994; 22: 441-7

Edwards SJ, Metheny NA. Measurement of gastric residual volume: state of the science. MEDSURG Nursing 2000; 9(3): 125-8

Engel JM, Muhling J, Junger A, Menges T, Karcher B, Hempelmann G. Enteral nutrition practice in a surgical intensive care unit: what proportion of energy expenditure is delivered enterally? Clin Nutr 2003 22(2): 187-92

Goodwin RS. Prevention of aspiration pneumonia: a research-based protocol. Dimens Crit Care Nurs 1996; 15: 58-71

Horn D, Chaboyer W, Schluter PJ. Gastric residual volumes in critically ill paediatric patients: a comparison of feeding regimens. Aust Crit Care 200417 (3): $98-100,102-3$ 
Jeejeebhoy KN. Short bowel syndrome: a nutritional and medical approach. CMAJ 2002166 (10): 1297-1302

Jeejeebhoy KN. Definition and mechanisms of diarrhoea. CMAJ 1977 116: 7379

Jostee Ca, Mustoe J, Collee G. Metoclopramide improves gastric motility in critical ill patients. Intens Care Med 1999; 25 (5): 464-9

Lewis SJ, Heaton KW. Stool form scale as a useful guide to intestinal transit time. Scan J Gastroenterol 199732 (9): 920-4

Lin HC, Van Citters GW. Stopping enteral feeding for arbitrary gastric residual volume may not be physiologically sound: results of a computer simulation model. J Parent Enteral Nutr 199721 (5): 286-9

Lukan J, McClave SA, Stefater A, Looney S, Matheson P, Gleeson K et al. Poor validity of residual volumes as a marker for aspiration. Am J Clin Nutr 2002; 75: 417S-8S

Mallampalli A, McClave SA, Snider HL. Defining tolerance to enteral feeding in the intensive care unit. Clin Nutr 200019 (4): 213-15 
Marshall AP, West SH. Enteral feeding in the critically ill: Are nursing practices contributing to hypocaloric feeding? Intensive Crit Care Nurs 2006; 22: 95-105 Mateo MA. Management of enteral tubes. Nursing management of enteral tube feeding. Heart Lung 1996; 25 (4): 318-23

McClave SA, Snider HL, Lowen CC, McLaughlin AJ, Greene LM, McCombs RG et al. Use of residual volume as a maker for enteral feeding intolerance: prospective blinded comparison with physical examination and radiographic findings. J Parent Enteral Nutr 1992; 16 (2): 99-105

McClave SA, Sexton LK, Spain DA, Admas JL, Owens NA, Sullins MB, et al. Enteral tube feeding in the intensive care unit : factors impeding adequate delivery. Crit Care Med 1999; 27 (7): 1252-6

McClave SA, DeMeo MT, DeLegge MH, DiSario A, Heyland DK, Maloney JP, et al. North American Submmit on aspiration in the critically ill patient: consensus statement. J Parent Enteral Nutr 2002; 26 (6 Suppl): S80-5

McClave SA, Snider HL. Clinical use of gastric residual volumes as a monitor for patients on enteral tube feedings. J Parent Enteral Nutr 200226 (6 Suppl): S43-8 
Metheny NA, Schallom ME, Edwards SJ. Effects of gastrointestinal motility and feeding tube site on aspiration risk in critically ill patients: a review. Heart Lung $2004 ; 33$ (3): 131-45

Metheny N. Minimizing respiratory complications of nasoenteric tube feedings: state of the science. Heart Lung 1993; 22: $213-23$

Mori S, Takemura CS, Yamaguchi I. Verificaçao do volume residual gástrico em unidade de terapia intensiva. Rev Bras Enferm 200356 (6):661-4

Murphy LM, Brickford V. Gastric residuals in tube feedings: how much is too much? Nutr in Clin Pract 1999; 14 (6): 304-6

Pingleton SK. Nutrition in chronic critical illness. Clin Chest Med 2001; 22 (1): $149-63$

Pinilla JC, Samphire J, Arnold C, Liu L, Thiessen B. Comparison of gastrointestinal tolerance to two enteral feeding protocols in critically ill patients: a prospective randomised controlled trial. J Parent Enteral Nutr 2001: 25 (2): 81-6

Powell KS, Marcuad SP, Farrior ES, Gallagher ML. Aspirating gastric residuals causes occlusion of small-bore feeding tubes. J Parent Enteral Nutr 1993 $17: 234-246$ 
Pullen RL. Measuring gastric residual volume. Nursing 2004 34: 18.

[ R Development Core Team. R: a language and environment for statistical computing. R Foundation for statistical computing 2006 ISBN 3-900051-07-0 ] Available at: http://www.r-project.org/ Last accessed: January, $9^{\text {th }} 2008$

Ramsay MA, Savege TM, Simpson BR, Goodwin R. Controlled sedation with alphaxalone_alphadolone. Br Med J 1974; 2: 656-59

Rayner CK, Samsom M, Jones KL, Horowitz M. Relationships of upper gastrointestinal motor and sensory function with glycemic control. Diabetes Care 2001 24(2) : 371-81

Ritz MA, Fraser R, Edwards N, Di Matteo AC, Chapman M, Butler R et al. Delayed gastric emptying in ventilated critically ill patients: measurement by 13 C-octanoic acid breath test. Crit Care Med 2001; 29: 1744-49

Samsom M, Akkermans LM, Jebbink RJ, van Isselt H, van-Berge-Henegouwen GP, Smout AJ. Gastrointestinal motor mechanisms in hyperglycaemia induced delayed gastric emptying in type I diabetes mellitus. Gut 199740 (5): 641- 46

Stechmiller KJ, Treloar D, Allen N. Gut dysfunction in critically ill patients: a review of the literature. Am J Crit Care 1997; 6: 204-9 
Steevens EC, Lipscomb AF, Poole GV, Sacks GS. Comparison of continuous vs intermittent nasogastric enteral feeding in trauma patients: perceptions and practice. Nutr Clin Pract 200217 (2): 118-22

Valls-Miró C, Carreño-Granero A, Domingo-Felici CM, Martinez-Estalella G, Llusà-Finestres J, Asensio-Malo G et al. Determinación del volumen gástrico residual: en busca de la mejor evidencia. Enfer Intensiva 2006; 17 (4): 154-62

Van der Voort P, Zandstra D. Enteral feeding in the critically ill: comparison between the supine and prone positions. A prospective crossover study in mechanically ventilated patients. Crit Care 20015 (4): 216-20

Williams TA, Leslie GD. A review of the nursing care of enteral feeding tubes in critically ill adults: part I. Intensive Crit Care Nurs 2004 20: 330-43

Williams TA, Leslie GD. A review of the nursing care of enteral feeding tubes in critically ill adults: part II. Intensive Crit Care Nurs 2005 21: 5-15

Zaloga GP. The myth of gastric residual volume. Crit Care Med 2005: 33 (2): $449-50$

Zhao J, Frokjaer JB, Drewes AM, Ejskjaer N. Upper gastrointestinal sensorymotor dysfunction in diabetes mellitus. World J Gastroenterol 200612 (18): 2846-57 


\section{Acknowledgements}

We thank the 2-1 ICU nursing staff of Bellvitge University Hospital, for their valuable assistance and participation. We are also grateful to the medical staff for their comments and collaboration.

We sincerely acknowledge the initial consultation with Dr. Josep Maria Argimon and his revision of the final version of the article.

We also thank so much to Dr. Rafael Mañez, Critical Care Medicine Chief

Officer and Dr. Ana Ochoa, Internal Medicine consultant for their critical appraisal of the project and this text.

\section{Conflict of interest statement}

This study was supported in part by a grant from Col.legi Oficial d'Infermeria de Barcelona, registered grant number 3063/2005.

The unique role of the study sponsors has been the evaluation of the project for assigning the financial support 